\title{
A DERIVATIVE FREE HIGH ORDERED HYBRID EQUATION SOLVER
}

\author{
Amit Kumar Maheshwari \\ Advanced Materials and Processes Research Institute (CSIR), Bhopal, India 462064
}

\begin{abstract}
Generally a range of equation solvers for estimating the solution of an equation contain the derivative of first or higher order. Such solvers are difficult to apply in the instances of complicated functional relationship. The equation solver proposed in this paper meant to solve many of the involved complicated problems and establishing a process tending towards a higher ordered by alloying the already proved conventional methods like Newton-Raphson method $(N-R)$, Regula Falsi method $(R-F) \&$ Bisection method $(B I S)$. The present method is good to solve those nonlinear and transcendental equations that cannot be solved by the basic algebra. Comparative analysis are also made with the other racing formulas of this group and the result shows that present method is faster than all such methods of the class.
\end{abstract}

\section{KEYWORDS}

Algebraic \& Non-Algebraic equations, Transcendental equations, Bisection method, Regula-Falsi method, Newton-Raphson method, Iteration Process.

\section{INTRODUCTION}

Many of the functional problem of type $f(x)=0$ appears in the theory of basic sciences for a perceptible solution. Generally, such equations appear in a convoluted mode possessing the partial and non-partial involvement of non algebraic and transcendental terms. Hence, they found difficulty in solving by the usual procedure of mathematics. In such cases, the Numerical methods are the only provision to deal such equations. The conventional methods like Bisection method, Regula falsi method and Newton-Raphson method are among the most general methods used for this purpose [1-6]. The various methods are discussed below.

\subsection{Bisection method}

This method is based on finding the root between two points of opposite ordinates. Here, we at first selects two points say, $x_{i}$ and $x_{i+1}$ such that their ordinate function gives a negative product value at these points i.e. $f\left(x_{i}\right) f\left(x_{i+1}\right)<0$. At each of the successive step, the new root can be 
calculated by a well defined mid-point say, $x_{m(i, i+1)}=\frac{x_{m(i)}+x_{m(i+1)}}{2}$ between them that causes the generation of two new intervals as follows,

$$
\begin{array}{ll}
\text { i. } & x_{m(i)} \text { and } x_{m(i, i+1)} \\
\text { ii. } & x_{m(i+1)} \text { and } x_{m(i, i+1)}
\end{array}
$$

Thus, there can be two cases

$$
f\left(x_{m(i)}\right) f\left(x_{m(i, i+1)}\right)=\left\{\begin{array}{l}
<0 \\
>0
\end{array} \text { For } i=1 \text { or } 2\right.
$$

The first case illustrates the existence of $m^{\text {th }}$ root between $x_{i}$ and $x_{m(i, i+1)}$ whereas the second case predicts the confinement of root between $x_{i+1}$ andc. For further modification, we take $f\left(x_{i}\right) f\left(x_{i+1}\right)<0$ to proceed further.

\subsection{Regula-Falsi method}

Regula-Falsi method is the improvement of Bisection method. The process algorithm interpreting the working rule is exactly the retrace of the path followed in Bisection method. However, the formula in present instances differences and follows the involvement of two ordinates which in particular for $x_{m(i, i+1)}$ can be represented as,

$$
x_{m(i, i+1)}=x_{i}-\frac{f\left(x_{i}\right)\left(x_{i+1}-x_{i}\right)}{f\left(x_{i+1}\right)-f\left(x_{i}\right)}
$$

\subsection{Newton-Raphson method}

Last two methods require the consideration of two points per calculating step. But, Newton Raphson method requires the single input point. Hence, the formula in present case is as follows,

$$
x_{i+1}=x_{i}-\frac{f\left(x_{i}\right)}{f^{\prime}\left(x_{i}\right)}
$$

This method requires the computation of single ordered derivative.

\subsection{Hybrid iteration $[7,8]$}

This method was originally proposed by He [7]. According to it, 


$$
x_{n+1}=\frac{-B \pm \sqrt{B^{2}-4 A\left(C+g\left(x_{n}\right)\right)}}{2 A}
$$

Later on, the evaluation of the complicacy in the result has been justified by Luo [8].

\subsection{Ide Modified Newton method of [9]}

It is the modified form of NR method whose equation runs as follows

$$
x_{n+1}=x_{n}-\left(\frac{4 f\left(x_{n}\right)}{f^{\prime}\left(x_{n}\right)+2 \frac{f\left(y_{n}\right)-f\left(x_{n}\right)}{y_{n}-x_{n}}+f^{\prime}\left(x^{N}{ }_{n+1}\right)}\right)
$$

\subsection{Maheshwari's Numerical Approach [10]}

The method has been developed by assuming the function $\mathrm{f}(\mathrm{X})$ to be continuous and differentiable in each point of its domain. The equation runs as follows,

$$
X=X_{0}-\frac{2 f\left(X_{0}\right)\left(X_{a}-X_{0}\right)}{\left[f\left(X_{a}\right)-f\left(X_{0}\right)\right]+f^{\prime}\left(X_{0}\right)\left(X_{a}-X_{0}\right)}
$$

\subsection{Maheshwari's Fourth order method of [11 ]}

The defining it is as follows,

$$
x_{n+1}=x_{n}+\frac{1}{f^{\prime}\left(x_{n}\right)}\left[\frac{\left\{f\left(x_{n}\right)\right\}^{2}}{\left\{f\left(y_{n}\right)-f\left(x_{n}\right)\right\}}-\frac{\left\{f\left(y_{n}\right)\right\}^{2}}{f\left(x_{n}\right)}\right]
$$

Inspite all the methods, many times it is quite difficult to obtain the derivative of a function due to its complicated nature. In those circumstances, it is quite difficult to use derivative based methods. The formulations illustrated through the present work is a target to handle such critical functional equations. Comparative analysis shows that the present method is faster than many methods of its class as shown in the example. 
International Journal of Information Technology, Modeling and Computing (IJITMC) Vol.1,No.4,November 2013

\section{FORMULA DERIVATION}

The general Taylor's series can be defined as,

$$
f(x+h)=\sum_{k=0}^{\infty} \frac{f^{(k)}(x)}{k !} h^{k}=f(x)+\frac{f^{\prime}(x)}{1 !} h+\frac{f^{\prime \prime}(x)}{2 !} h^{2}+\cdots+\frac{f^{(k)}(x)}{k !} h^{k}+o\left(h^{k+1}\right)(7)
$$

Hence about the point $x_{m 0}$

$$
f(x)=f\left(x_{m 0}\right)+\frac{f^{\prime}(x)}{1 !}\left(x-x_{m 0}\right)+\frac{f^{\prime \prime}(x)}{2 !}\left(x-x_{m 0}\right)^{2}+\frac{f^{\prime \prime \prime}(x)}{3 !}\left(x-x_{m 0}\right)^{3} \cdots+\frac{f^{(k)}(x)}{k !}\left(x-x_{m 0}\right)^{k}+o\left(\left(x-x_{m 0}\right)^{k+1}\right)
$$

If $f(x)=0$ is the given algebraic equation containing transcendental terms, the truncation to two terms of this expression lead to,

$$
f(x)=f\left(x_{m 0}\right)+\frac{f^{\prime}(x)}{1 !}\left(x-x_{m 0}\right)+\frac{f^{\prime \prime}(x)}{2 !}\left(x-x_{m 0}\right)^{2}+o\left(h^{3}\right)=0
$$

Considering this quadratic nature of the expression, we can find the solution as,

$$
\left(x-x_{m 0}\right)=\frac{-f^{\prime}(x) \pm \sqrt{\left(f^{\prime}(x)\right)^{2}-2 f^{\prime \prime}(x) f\left(x_{0}\right)}}{f^{\prime \prime}(x)}
$$

This can be alternatively written as,

$$
x=x_{m 0}+\frac{-f^{\prime}(x) \pm \sqrt{\left(f^{\prime}(x)\right)^{2}-2 f^{\prime \prime}(x) f\left(x_{0}\right)}}{f^{\prime \prime}(x)}
$$

However, we can use the conventional methods to remove the derivatives through a simple divided difference function " $\mathrm{D}$ " to obtain the following expression,

$$
x=x_{m 0}+\frac{f_{R F}-f_{N R} \pm \sqrt{\left(f_{N R}-f_{R F}\right)^{2}-2\left(x_{N R}-x_{R F}\right)\left(D f_{N R, R F}-D f_{B I S, R F}\right) f\left(x_{0}\right)}}{\left(D f_{N R, R F}-D f_{B I S, R F}\right)}
$$




\section{PROCESS ALGORITHM}

The process algorithm in the present case runs through the following steps.

i) Choose any two points say $X_{0}$ and $X_{1}$ such that $f\left(X_{m 0}\right)$ and $f\left(X_{m l}\right)$ are opposite in sign. The ordinate with lesser $f(X)$ is assigned $X_{m 0}$.

ii) Find the results by Bisection method, Regula Falsi method and the present formula for the two points $X_{n 0}$ and $X_{n l}$. However, the square root may be neglected in those circumstances where an imaginary condition falls.

iii) Further iteration steps were processed by assigning $X_{(m+1) 0}$ as the result of NewtonRaphson formula \& $X_{(m+1) l}$ as the Result of present formula for the corresponding $m^{\text {th }}$ step.

iv) The process is repeated to get the result from next iteration step which in turn will give more exact result.

v) The repetition of the above process is terminated on obtaining a required level of accuracy.

\section{NUMERICAL RESULTS AND DISCUSSIONS}

Following illustrative problems shows the result obtained by present method to solve the said equations. At the same time, these results are compared with other conventional and recently proposed methods by other mathematicians to compare the efficiency and accuracy of it.

Illustrative problem: Consider a equation $[8,9,11]$

$$
f(x)=\sin x=0
$$

Various results for Newton iteration, Hybrid iteration, Modified Newton method of Nasr-Al-Din Ide, Maheshwari's method and present iteration method in present case of illustrative example are shown in Tables $4-8$.

$$
\text { Table 1: Newton iteration [8] for solving } f(x)=\sin x=0
$$

\begin{tabular}{lll}
\hline$n$ & $x_{n}$ & $\left|f\left(x_{n}\right)\right|$ \\
\hline 1 & -12.601419947171719 & $0.03504215716101725900(3.5 \mathrm{E}-2)$ \\
2 & -12.566356255118672 & $0.00001435924050063514(1.4 \mathrm{E}-5)$ \\
\hline
\end{tabular}


International Journal of Information Technology, Modeling and Computing (IJITMC) Vol.1,No.4,November 2013

Table 2: Hybrid iteration [9] for solving $f(x)=\sin x=0$

\begin{tabular}{lll}
\hline$n$ & $x_{n}$ & $\left|f\left(x_{n}\right)\right|$ \\
\hline 1 & 2.9869052804082314 & 0.15407121285051845000 \\
2 & 3.2915361735771942 & 0.14938228643233631000 \\
3 & 3.1467836457377532 & $0.005190968834899997360(5.2 \mathrm{E}-3)$ \\
4 & 3.1410906419036979 & $0.00050201166500952066(5.0 \mathrm{E}-4)$ \\
5 & 3.1415926228169786 & $0.00000003077281464126(3.1 \mathrm{E}-8)$ \\
6 & 3.1415926525599063 & $0.00000000102988696493(1.0 \mathrm{E}-9)$ \\
7 & 3.1415926545974751 & $0.00000000100768181543(1.0 \mathrm{E}-9)$ \\
8 & 3.1415926576620539 & $0.00000000407226062837(4.1 \mathrm{E}-9)$ \\
9 & 3.1415926674045491 & $0.00000001381475588335(1.4 \mathrm{E}-8)$ \\
10 & 3.1415926662849540 & $0.00000001269516080004(1.3 \mathrm{E}-8)$ \\
11 & 3.1415926605950051 & $0.00000000700521184360(7.0 \mathrm{E}-9)$ \\
12 & 3.1415926640530745 & $0.00000001046328123000(1.0 \mathrm{E}-8)$ \\
13 & 3.1415926498831088 & $0.00000000370668441530(3.7 \mathrm{E}-9)$ \\
14 & 3.1415926445379925 & $0.00000000905180076483(9.1 \mathrm{E}-9)$ \\
15 & 3.1415926440528610 & $0.00000000953693225536(9.5 \mathrm{E}-9)$ \\
16 & 3.1415926443032149 & $0.00000000928657829558(9.3 \mathrm{E}-9)$ \\
17 & 3.1415926408815600 & $0.00000001270823325495(1.3 \mathrm{E}-8)$ \\
\hline
\end{tabular}

Table 3: Nasr-Al-Din Ide [12] for solving $f(x)=\sin x=0$

\begin{tabular}{lll}
\hline$n$ & $x_{n}$ & $\left|f\left(x_{n}\right)\right|$ \\
\hline 1 & -1.9296287714411738020 & 0.9363074853509102 \\
2 & -3.7534514114768829580 & 0.57438999688181760 \\
3 & -3.1183795738554536810 & 0.0232109950747530 \\
\hline
\end{tabular}

Table 4: Maheshwari's Method [10] for solving $f(x)=\sin x=0$

\begin{tabular}{lcc}
\hline$n$ & $x_{n}$ & $\left|f\left(x_{n}\right)\right|$ \\
1 & 0.319035098 & 0.31365 \\
2 & -0.091342606 & 0.09122 \\
3 & -0.004965930 & 0.00497 \\
4 & 0.000348994 & 0.00035
\end{tabular}

Table 5: Maheshwari's Fourth order method [11] for solving $f(x)=\sin x=0$

\begin{tabular}{lll}
\hline$n$ & $x_{n}$ & $\left|f\left(x_{n}\right)\right|$ \\
\hline 1 & -12.14025012250240000 & 0.413341334176536000 \\
2 & -12.56762318740160000 & 0.001252572714843290 \\
\hline
\end{tabular}


Table 6: Present Method for solving $f(x)=\sin x=0$

\begin{tabular}{ccc}
\hline$n$ & $x_{n}$ & $\left|f\left(x_{n}\right)\right|$ \\
\hline 1 & 3,13550745968220000000000 & $0,0060851563522972400000(6.1 \mathrm{E}-3)$ \\
2 & 3,1415912646160400000000 & $0,0000013889737570215700(1.4 \mathrm{E}-6)$ \\
\hline
\end{tabular}

Other Illustrative problems: Other example includes,

Table 7: Comparison of the result for the functions $f_{1}(x)$ to $f_{4}(x)$

\begin{tabular}{|c|c|c|c|c|}
\hline Function & Conditions & $\begin{array}{c}\text { Solution by present } \\
\text { method }\end{array}$ & $\begin{array}{c}\text { Number of } \\
\text { Iterations }\end{array}$ & Error \\
\hline$f_{1}(x)=e^{x}-3 x^{2}=0$ & $x_{0}=1, x_{1}=0.5$ & 0,9100076138792720 & 3 & $-1,23166 \mathrm{E}-07$ \\
\hline$f_{2}(x)=x^{2}-(1-x)^{5}=0$ & $x_{0}=0.5, x_{1}=0.2$ & 0,3459548128842710 & 4 & $-4,76271 \mathrm{E}-09$ \\
\hline$f_{3}(x)=x^{3}-e^{-*}=0$ & $x_{0}=0.5, x_{1}=1$ & 0,7728829656002800 & 4 & $1,45389 \mathrm{E}-08$ \\
\hline$f_{4}(x)=x^{3}-2 x-5=0$ & $x_{0}=2, x_{1}=2.5$ & 2,0945514862141200 & 3 & $5,21439 \mathrm{E}-08$ \\
\hline
\end{tabular}

\section{REFERENCES}

[1] Curtis F. Gerald, Patrick O. Wheatley (2004), Applied Numerical Analysis, Pearson Addition Wesley, New York, seventh edition.

[2] Hildebrand F.B. (1982), Introduction to Numerical Analysis, Tata Mc. Graw, Hill Publishing Co. limited, New Delhi, Second Edition.

[3] James M. Ortega, William G. Poole, Jr. (1981), An Introduction to Numerical methods for differential Equations, Pitman Publishing Inc., Massachussets.

[4] Kreyszig Ervin (1972), Advanced Engineering Mathematics, Willey Eastern Limited, New Delhi, Third U.S. Edition.

[5] Sidi Avram (2006), Unified Treatment of Regula Falsi, Newton-Raphson, Secant, and Steffensen Methods for Nonlinear Equations, Journal of Online Mathematics and Its Applications, 1-13.

[6] Terry E. Shoup (1984), Applied Numerical methods for the microcomputer, Printice - Hall Inc., New Jersey.

[7] He J.H. (2003) A new iteration method for solving algebraic equations. Applied Mathematics and Computation, $135(1), 81-84$.

[8] Luo Xing-Guo (2005). A note on the new iteration method for solving algebraic equation. Applied Mathematics and Computation, 171 (2), 1177-1183.

[9] Ide Nasr-Al-Din (2008), On modified Newton methods for solving a non linear algebraic equations, Applied Mathematics and Computation, 198, 138-142.

[10] Maheshwari Amit Kumar (2012). Solution of Algebraic and Transcendental Equation: A New Numerical Approach. Anushandhan 1(1), 62-65.

[11] Maheshwari Amit Kumar (2009), A fourth order iterative method for solving nonlinear equations, Applied Mathematics and Computation, 211, 383-391. 